\section{FLOW INJECTION VOLTAMMETRIC MULTI-SITE DETECTION POTENTIALITIES}

Rita I.L. Catarino, M.B. Quinaz Garcia* e José L.F.C. Lima

CEQUP/ Departamento de Química-Física, Faculdade de Farmácia, Universidade do Porto,

R. Anibal Cunha, 164, 4050 Porto, Portugal

\section{Abstract}

This paper describes the evaluation of a flow injection system with voltammetric multi-site detection, which enables the detector to carry out the measure of the sample at different positions in the manifold. Some advantages of these systems are partially discussed.

A voltammetric cell with a tubular configuration and small inner volume $(105 \mu 1)$ is proposed. This configuration has a very reduced effect on the hydrodynamic characteristics of the flow which makes it most attractive for manifolds with sequential detection.

The manifold optimization was carried out using potassium hexacyanoferrate (II) in order to achieve a good separation of both analytical signals, a minimal sample dispersion and a high sampling rate.

Key words: FIA; multi-site detection; voltammetry; tubular electrodes.

\section{Introdução}

A análise por injeç̧ão em fluxo (FIA) [1] devido às suas vantagens tais como simplicidade, baixo custo, reduzido consumo de reagentes e versatilidade tem-se mostrado de grande utilidade em análises de rotina, abrangendo campos tão variados como a análise ambiental, clínica ou farmacêutica. Tais características facilitam a adaptação dos sistemas FIA a diferentes tipos de detectores e diferentes técnicas, requerendo somente pequenas modificações na sua configuração.

Os primeiros sistemas FIA desenvolvidos, os quais apresentavam uma enorme simplicidade rapidamente deram lugar a sistemas um pouco mais complexos que possibilitam a realização de mais do que uma determinação do mesmo segmento de amostra.

A multideteç̧ão pode ser realizada apenas com um detector, pelo qual o segmento de amostra flui repetidas vezes [2], ou recorrendo a vários detectores colocados estrategicamente ao longo da montagem [3]. Todos estes sistemas têm em comum o facto de os detectores ocuparem uma posição fixa.

autor para quem a correspondência deve ser dirigida
Recentemente o nosso grupo de investigação em trabalho conjunto com o Centro de Energia Nuclear na Agricultura (Brasil) sugeriu o conceito de detecção multilocalizada em FIA [4], o qual confere mobilidade ao detector. Este novo conceito tem por base a recolocação de um único detector em várias posições ao longo de uma montagem permitindo quer a monitorização sequencial, quer a monitorização em paralelo [5].

A utilidade analítica do uso de sistemas FIA com detecção multilocalizada foi evidenciada através de vários trabalhos envolvendo quer detectores espectrofotométricos [4-6] quer potenciométricos [7]

A voltametria nas suas diversas variantes é um método de análise com grandes potencialidades, nomeadamente pelas baixas concentrações que permite quantificar, pela elevada selectividade que possui, a qual pode ser exaltada pela simples modificação de algumas variáveis electroquímicas e pelo baixo custo comparativamente a outras técnicas de análise, nomeadamente a espectrofotometria de absorção atómica com atomização electrotérmica.

Apesar destas vantagens as técnicas voltamétricas têm vindo a ser preteridas nas determinações de rotina laboratorial dado que o processamento das amostras, e, muitas vezes o condicionamento dos eléctrodos associado a este tipo de determinações é demasiado lento e desajustado aos sistemas automáticos de fluxo [8].

É nossa conviç̧ão que muitas destas dificuldades podem ser ultrapassadas combinando os detectores voltamétricos com sistemas de fluxo que lhes confiram mobilidade.

Assim o acoplamento do conceito de deteç̧ão multilocalizada com detectores voltamétricos apresenta várias vantagens: torna viável a realização de determinações sequenciais de espécies na mesma amostra ou ensaios de especiação, permite efectuar medições com paragem de fluxo, facilita o condicionamento químico da superfície do eléctrodo de trabalho e viabiliza a automatização de métodos de adição.

Apesar das referidas vantagens, os resultados obtidos com este tipo de sistemas podem ser comprometidos quer pelo volume interno da célula detectora [4], quer pela sua configuração visto ser essencial a manutenção das características hidrodinâmicas do segmento de amostra durante a deteç̧ão $[4,5]$. Estes condicionalismos impuseram-nos a construção de uma célula detectora de configuração tubular e volume interno reduzido por forma a retirar o máximo de vantagens da detecção voltamétrica multilocalizada.

O objectivo do trabalho desenvolvido foi o de avaliar o comportamento da referida célula electroquímica quando incorporada num sistema FIA com monitorização voltamétrica sequencial.

Realizaram-se vários estudos envolvendo a oxidação do hexacianoferrato de potássio (II), os quais conduziram à optimização do sistema tendo como objectivos: a obtenção de uma eficaz separação dos sinais analíticos medidos nas duas posições de detecção, a minimização da dispersão 
do segmento de amostra ao longo do reactor que separa os dois locais de detecção e a obtenção de um elevado ritmo de amostragem.

\section{Parte Experimenta}

Reagentes

Foram utilizados reagentes de qualidade p.a. ou semelhante e água purificada por sistema Millipore Milli Q (condutividade específica $<0,1 \mu \mathrm{S} . \mathrm{cm}^{-1}$ ) na preparação de todas as soluções e nas diluições das amostras.

Como solução transportadora foi utilizada uma solução de $\mathrm{KCl}$ 0,1 M.

As soluções padrão de $\mathrm{K}_{4}\left[\mathrm{Fe}(\mathrm{CN})_{6}\right]$, com concentrações compreendidas entre $1 \times 10^{-5} \mathrm{e} 1 \times 10^{-3}$ $M$ foram preparadas diariamente por diluições sucessivas a partir de uma solução de $\mathrm{K}_{4}\left[\mathrm{Fe}(\mathrm{CN})_{6}\right]$ com concentração $1 \times 10^{-3} \mathrm{M}$.

\section{Aparelhagem e eléctrodos}

No sistema FIA desenvolvido (Fig.1 - A), as soluções foram impulsionadas por uma bomba peristáltica Gilson Miniplus 3, e a intercalação dos segmentos de amostra na solução portadora foi efectuada através de um injector-comutador de movimentação circular e com funcionamento idêntico ao anteriormente referenciado com movimentação horizontal [9], o qual simultaneamente permitia o reposicionamento do detector ao longo da montagem [4].

A ligação entre os diferentes componentes do sistema foi efectuada com tubos de Teflon da marca Omnifit de diâmetro interno 0,8 mm. Algumas peças de ligação auxiliares, nomeadamente o suporte do eléctrodo de referência foram construídas e usadas como previamente descrito [10].

A célula electroquímica utilizada consistia no seguinte conjunto de três eléctrodos: eléctrodo de trabalho de grafite prensado, eléctrodo de referência de $\mathrm{AgCl} / \mathrm{Ag}(3,0 \mathrm{M}, \mathrm{KCl})$ e eléctrodo auxiliar de grafite prensado. Quer o eléctrodo de trabalho, quer o eléctrodo auxiliar apresentavam configuração tubular e foram construídos dissolvendo $0,25 \mathrm{~g}$ de parafina sólida em cerca de $10 \mathrm{ml}$ de n-hexano a $40^{\circ} \mathrm{C}$. Posteriormente adicionou-se $4,75 \mathrm{~g}$ de pó de grafite e continuou-se o aquecimento, com agitação, até completa evaporação do solvente orgânico. Pesou-se $0,20 \mathrm{~g}$ do sólido assim obtido e comprimiu-se (pressão de $19,000 \mathrm{Kg} / \mathrm{cm}^{2}$ ) num molde construído com material capaz de suportar altas pressões. Foram obtidos discos de diâmetro $10 \mathrm{~mm}$ e cerca de 1,2 $\mathrm{mm}$ de espessura. Uma porção do disco obtido, após execução do contacto eléctrico, foi colocada num molde de perspex apropriado segundo o processo já referido anteriormente na construção de detectores potenciométricos tubulares de membrana cristalina homogénea [11].

O eléctrodo de referência de $\mathrm{AgCl} / \mathrm{Ag}$ foi colocado perpendicularmente ao sentido do fluxo, por forma a minimizar o volume interno da célula electroquímica (Fig.1 - B). O volume interno da célula detectora e respectivos acessórios foi avaliado em aproximadamente $105 \mu \mathrm{l}$, mediante a titulação do anião cloreto recolhido do interior do "loop" da célula detectora pelo catião prata (n=6). A recolha do anião cloreto foi executada fazendo passar uma solução de cloreto de potássio pela célula detectora, a qual foi posteriormente deslocada para um outro canal por forma a que a solução nela contida fosse arrastada por uma corrente de ar e recolhida num goblé.

As determinações voltamétricas e amperométricas foram efectuadas num sistema electroquímico da marca Ecochimie/ Autolab modelo PSTAT 10.

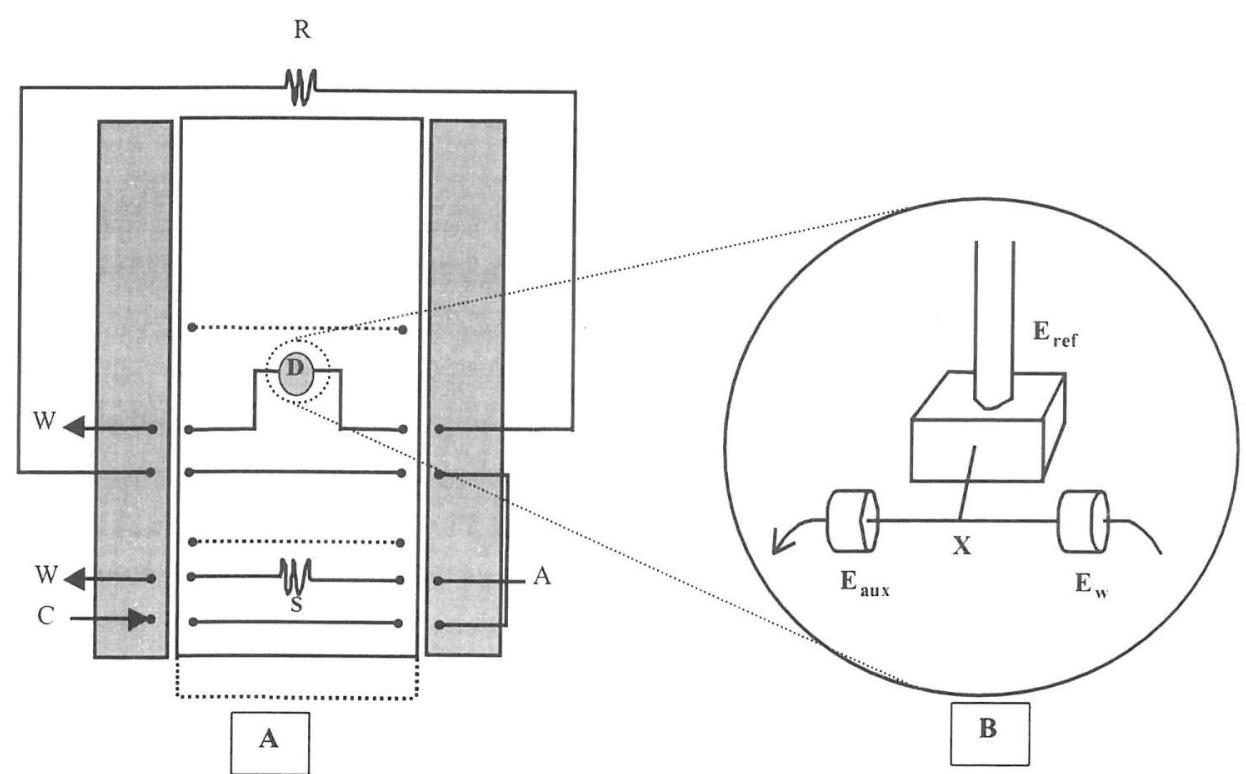

Figura.1 - A - Representação esquemática do sistema FIA com monitorização sequencial desenvolvido: A - amostra (Q); C - solução portadora - $\mathrm{KCl}$ 0,1 M (Q); D - detector; R - reactor (L); S - "loop" de amostra (V); W - dreno; $\rightarrow$ locais onde foi aplicado o sistema de impulsão. B - $E_{\text {aux }}$ - eléctrodo auxiliar; $\mathrm{E}_{\text {ref }}$ - eléctrodo de referência; $E_{\mathrm{w}}$ - eléctrodo de trabalho; $\mathrm{X}$ confluência. 


\section{Resultados e Discussão}

A célula electroquímica desenvolvida (Fig.1 - B) foi, numa primeira fase avaliada num sistema de FIA monocanal de baixa dispersão no qual ocupava uma posição fixa. Após obtenção de resultados reveladores de elevada precisão $(\mathrm{CV} \%=0,9, \mathrm{n}=12)$ a célula electroquímica foi incorporada numa montagem FIA com deteç̧ão multilocalizada sequencial. Procedeu-se então ao estudo de vários parâmetros da montagem tendo em vista a sua optimização.

A configuração tubular do detector é a mais adequada visto não alterar significativamente as características hidrodinâmicas do fluxo devido ao diâmetro interno da célula detectora $-0,8 \mathrm{~mm}-$ estar em consonância com o diâmetro interno da tubagem do sistema. O mesmo não se verifica com outros tipos de células, nomeadamente as de jacto impingente e as de camada fina, as quais alteram as características hidrodinâmicas do fluxo comprometendo a medição do segmento de amostra no segundo local de detecção. Quanto ao volume do "loop" da célula detectora $(105 \mu 1)$ pode considerar-se satisfatório, não comprometendo a obtenção de ritmos de amostragem elevados.

Dado que quando o detector se move toda a solução que retem é transportada para a sua nova posição é possível o aparecimento de problemas inerentes a diferenças entre as correntes de fluxo associadas a ambas as posições de deteç̧ão (nomeadamente diferenças de concentrações de electrólitos ou pH, entre outras). Também por esta razão é importante que o volume da célula detectora e respectivos acessórios seja o menor possível.

Um parâmetro que influencia significativamente o grau de dispersão do segmento de amostra que ocorre entre as duas posições de deteç̧ão é o comprimento do reactor localizado entre os dois pontos de detecção [4]. Este valor foi optimizado em três metros visto que para valores menores e atendendo aos outros parâmetros (caudal, volume de amostra e tempos de comutação), não se obtinha uma eficaz separação dos sinais analíticos; para valores superiores o grau de dispersão do segmento de amostra era maior, comprometendo os valores do limite de detecção das determinações realizadas no segundo ponto de detecção.

De referir que o tempo de comutação foi um parâmetro importante que também se avaliou, dado que a injecção do segmento de amostra ocorre simultaneamente com o reposicionamento do detector. Tal facto conduz à possibilidade de ocorrência de sobreposição de segmentos de amostra se os tempos de comutação não forem os mais ajustados, podendo conduzir a uma ineficaz separação dos dois sinais analíticos medidos [4]

Os intervalos de valores das variáveis estudados durante a optimização da referida montagem estão apresentados na Tabela 1.
Tabela 1 - Parâmetros avaliados durante a optimização do sistema

\begin{tabular}{cccccccc}
\hline & $\begin{array}{c}\text { Volume de } \\
\text { Amostra }(\mu \mathrm{l})\end{array}$ & $\begin{array}{c}\text { Caudal } \\
(\mathrm{ml} / \mathrm{min})\end{array}$ & $\begin{array}{c}\text { Comprimento } \\
\text { do reactor (m) }\end{array}$ & Potencial (V) & $\mathbf{K C l}(\mathbf{M}) \begin{array}{c}\text { Tempo de } \\
\text { comutação (s) } \\
1^{\mathbf{a}} \text { posição 2 }\end{array}$ 20sição \\
\hline $\begin{array}{c}\text { Intervalo de valores } \\
\text { ensaiados }\end{array}$ & $50-200$ & $0,8-2,0$ & $2-5$ & $0,2-0,9$ & $0,1-1,0$ & $20-90$ \\
\hline $\begin{array}{c}\text { Valor } \\
\text { optimizado }\end{array}$ & 100 & 1,2 & 3 & 0,5 & 0,1 & 30 & 55 \\
\hline
\end{tabular}

O sistema proposto apresentou uma boa precisão em ambas as posições de detecção com coeficientes de variação (CV\%) de, respectivamente 1,3 e 1,8\% (n=12) - Fig.2. As rectas de calibração para uma gama de concentrações compreendida entre $1 \times 10^{-5} \mathrm{e} 1 \times 10^{-3} \mathrm{M}$ apresentaram um coeficiente de correlação superior a 0,995. Quanto ao ritmo de amostragem foi de 45 amostras por hora, o que tratando-se de um sistema com duas medidas sequenciais corresponde a 90 determinações por hora.

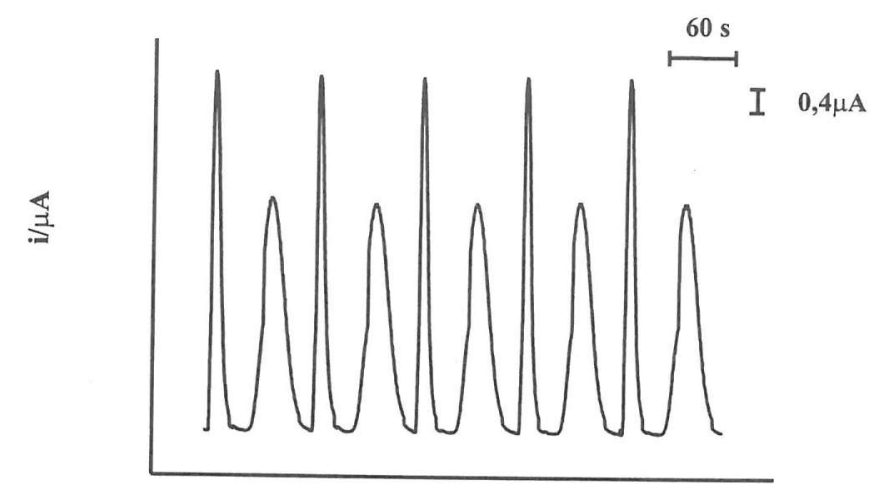

$\mathrm{t} / \mathrm{s}$

Figura.2 - Registo correspondente à determinação sequencial de $\mathrm{K}_{4}\left[\mathrm{Fe}(\mathrm{CN})_{6}\right]$. 


\section{Conclusões}

O estudo desenvolvido permitiu demonstrar as potencialidades destes eléctrodos tubulares na detecção voltamétrica multilocalizada, abrindo várias perspectivas de aplicações futuras, nomeadamente em estudos envolvendo amostras que apresentem vantagens na sua determinação sequencial, caso dos estudos de especiação e dos estudos cinéticos

É de salientar que a preparação destes detectores voltamétricos tubulares é suficientemente flexivel para se pensar na adaptação deste processo de construção ao desenvolvimento de eléctrodos tubulares de outros materiais (por exemplo de carbono vítreo ou ouro).

\section{Agradecimentos}

Os autores agradecem o financiamento obtido através do projecto PRAXIS/C/QUI/10035/1998. Um de nós (R.I.L.C.) agradece ao programa PRAXIS XXI a sua bolsa de doutoramento (BD/13843/97).

\section{Referências}

1 - J. Ruzicka e E.H. Hansen, Anal. Chim. Acta, 99, (1978), 37-76;

2 - A. Rios, M.D. Luque de Castro e M. Valcárcel, Anal. Chim. Acta, 179, (1986), 463-468;

3 - J.H. Dahl, D. Espersen e A. Jensen, Anal. Chim. Acta, 105, (1979) 327-333;

4 - E.A.G. Zagatto, H. Bergamin Fo, S.M.B. Brienza, M.A.Z. Arruda, A.R.A. Nogueira e J.L.F.C. Lima, Anal. Chim. Acta, 261, (1992), 59-65;

5 - A.R.A. Nogueira, S.M.B. Brienza, E.A.G. Zagatto, J.L.F.C. Lima e A.N. Araújo, Anal. Chim. Acta, 276, (1993), 121-125;

6 - A.N. Araújo, J.A.M. Catita, J.L.F.C. Lima e E.A.G. Zagatto, Fresenius J. Anal. Chem., 360, (1998), 100-103;
7 - J.A.G. Neto, A.R.A. Nogueira, H. Bergamin F ${ }^{0}$, E.A.G. Zagatto, J.L.F.C. Lima e M.C.B.S.M. Montenegro, Anal. Chim. Acta, 285, (1994), 293-299;

8 - L. Taylor, Current Separations, 16:2, (1997), 314-319;

9 - F.J. Krug, H. Bergamin Fo e E.A.G. Zagatto, Anal. Chim. Acta, 179, (1986), 103-118;

10 - J. Alonso, J. Bartroli, J.L.F.C. Lima e A.A.S.C. Machado, Anal. Chim. Acta, 179, (1986), 503 508

11 - I.M.P.L.V.O. Ferreira, J.L.F.C. Lima e L.S.M. Rocha, Fresenius J. Anal. Chem., 347, (1993), 314-319;

Received. August 14, 1999 Revised. Septembre 11. 1999 